\title{
Development of a Non-Iterative Balance Load Prediction Algorithm for the NASA Ames Unitary Plan Wind Tunnel
}

\author{
N. Ulbrich ${ }^{\dagger}$ \\ Jacobs Technology Inc., Moffett Field, California 94035 \\ M. Amaya \\ NASA Ames Research Center, Moffett Field, California 94035 \\ A. L'Esperance ${ }^{\S}$ \\ NASA Ames Research Center, Moffett Field, California 94035
}

\begin{abstract}
A non-iterative load prediction algorithm for strain-gage balances was developed for the NASA Ames Unitary Plan Wind Tunnel that computes balance loads from the electrical outputs of the balance bridges and a set of state variables. A state variable could be, for example, a balance temperature difference or the bellows pressure of a flow-through balance. The algorithm directly uses regression models of the balance loads for the load prediction that were obtained by applying global regression analysis to balance calibration data. This choice greatly simplifies both implementation and use of the load prediction process for complex balance configurations as no load iteration needs to be performed. The regression model of a balance load is constructed by using terms from a total of nine term groups. Four term groups are derived from a Taylor series expansion of the relationship between the load, gage outputs, and state variables. The remaining five term groups are defined by using absolute values of the gage outputs and state variables. Terms from these groups should only be included in the regression model if calibration data from a balance with known bi-directional outputs is analyzed. It is illustrated in detail how global regression analysis may be applied to obtain the coefficients of the chosen regression model of a load component assuming that no linear or massive near-linear dependencies between the regression model terms exist. Data from the machine calibration of a six-component force balance is used to illustrate that the accuracy of the non-iterative load prediction algorithm is as good as the accuracy of the alternate iterative load prediction algorithm.
\end{abstract}

\section{Nomenclature}

A $=$ matrix that is needed for the definition of a global regression analysis problem

$a_{i, 0}, a_{i, 1}, a_{i, 2}, \ldots \quad=$ coefficients of the regression model of a single load component $F_{i}$

$A F \quad=$ axial force

B $\quad$ = row vector that is needed for the balance load prediction

b = vector that is needed for the definition of a global regression analysis problem

D $\quad$ = vector of the independent variables of a balance load component

$D_{j} \quad=$ independent variable value or gage output difference with index $j$

$\mathbf{F} \quad=$ vector of balance load components

$\dagger$ Aerodynamicist, Jacobs Technology Inc.

$\ddagger$ Data Engineer, Code AOO, NASA Ames Research Center

$\S$ Test Engineer, Code AOO, NASA Ames Research Center 


\begin{tabular}{|c|c|}
\hline$F$ & $=$ single load component of a strain-gage balance \\
\hline$F_{i}$ & $=$ load component with index $i$ \\
\hline$F_{j}$ & $=$ load component with index $j$ \\
\hline$i$ & $=$ index \\
\hline$j$ & $=$ index \\
\hline$k$ & $=$ index \\
\hline$L$ & $=$ auxiliary variable \\
\hline$m$ & $=$ total number of independent variables of a balance load component \\
\hline$n$ & $=$ total number of load components of a balance \\
\hline$N_{j}$ & $=$ natural zero output of a balance gage with index $j$ \\
\hline$N 1$ & $=$ forward normal force of a force balance \\
\hline$N 2$ & $=$ aft normal force of a force balance \\
\hline$p$ & $=$ number of data points of a balance calibration data set \\
\hline$r A F$ & $=$ electrical output of axial force gage \\
\hline$r F_{j}$ & $=$ electrical output of a generic balance gage \\
\hline$r N 1$ & $=$ electrical output of the forward normal force gage of a force balance \\
\hline$r N 2$ & $=$ electrical output of the aft normal force gage of a force balance \\
\hline$r R M$ & $=$ electrical output of the rolling moment gage of a force balance \\
\hline$r S 1$ & $=$ electrical output of the forward side force gage of a force balance \\
\hline$r S 2$ & $=$ electrical output of the aft side force gage of a force balance \\
\hline$T$ & $=$ temperature of the balance \\
\hline$T_{\circ}$ & $=$ reference temperature of the balance \\
\hline$R M$ & $=$ rolling moment of a force balance \\
\hline$S_{\nu}$ & $=$ sum of all terms of a regression model term group with index $\nu$ \\
\hline$S 1$ & $=$ forward side force of a force balance \\
\hline$S 2$ & $=$ aft side force of a force balance \\
\hline $\mathbf{x}$ & $=$ vector of a global regression analysis problem that stores the regression coefficients \\
\hline $\mathbf{y}$ & $=$ auxiliary vector that stores regression coefficients \\
\hline $\mathbf{z}$ & $=$ rectangular matrix that stores regression coefficients \\
\hline$\alpha_{i, j}$ & $=$ regression coefficient \\
\hline$\beta_{i, j}$ & $=$ regression coefficient \\
\hline$\gamma_{i, j}$ & $=$ regression coefficient \\
\hline$\delta$ & $=$ number of regression model terms that the balance calibration data supports \\
\hline$\delta_{i j}$ & $=$ Kronecker delta \\
\hline$\Delta N 1$ & $=$ load residuals of the forward normal force \\
\hline$\lambda$ & $=$ total number of possible regression model terms \\
\hline$\mu_{\nu}$ & $=$ number of possible terms of a regression model term group \\
\hline$\nu$ & $=$ regression model term group index \\
\hline$\xi$ & $=$ regression coefficient index \\
\hline$\varphi(i, j)$ & $=$ summation term of a double sum that depends on two summation indices \\
\hline$\psi$ & $=$ upper bound of a summation index \\
\hline
\end{tabular}

\section{Introduction}

Measurements of the electrical outputs of balance bridges are traditionally used to predict the loads, i.e., the forces and moments, that act on an $n$-component strain-gage balance during a wind tunnel test. However, balance loads can also be a function of additional state variables that may have to be used as input for the load prediction. For example, it is known in the aerospace testing community that a temperature difference between balance calibration laboratory and wind tunnel can cause primary gage sensitivity shifts. These shifts may be predicted if the balance is calibrated at different temperature levels (see, e.g., results reported in Ref. [1]). Then, the difference between the balance temperature and a reference temperature may become an input variable for the load prediction. In addition, tests of aircraft and spacecraft with 
propulsion simulators are sometimes conducted in wind tunnel facilities. These simulators are often powered by high-pressure air that is routed through a so-called "flow-through balance" from the model support system to exhaust nozzles on the model itself. Both calibration and use of a "flow-through balance" is complex as the unavoidable bridging of the metric part of the balance introduces an internal pressure, i.e., the so-called "bellows pressure," as an additional input variable for the balance load prediction.

Different approaches are used in the aerospace testing community to predict balance loads from measured outputs during a wind tunnel test. The load prediction process of all these approaches is obtained from a multivariate regression analysis of balance calibration data. The Iterative Method, for example, fits the electrical outputs of the balance as a function of the loads and, afterwards, constructs an iteration scheme from the result so that loads can be predicted from outputs (see Ref. [2] for a detailed description of this approach). The use of a load iteration scheme, however, is only possible if the number of balance loads exactly matches the number of other variables, e.g., electrical outputs of the strain-gages, that are needed as input for the load prediction. This requirement is violated if either a state variable like the temperature difference or the bellows pressure is needed as input for the load prediction. Therefore, an approach was developed a few years ago at NASA Ames Research Center that introduces the state variable as both an independent and dependent variable. This approach makes it possible to construct an iteration scheme for the load prediction (see Ref. [3] for a detailed description of the approach).

An alternate approach exists for the prediction of balance loads that also fits balance calibration data but avoids the use of a load iteration equation. This approach is called the Non-Iterative Method. It directly fits each balance load component as a function of the measured electrical outputs and state variables (see Ref. [4] for a description of the approach). Consequently, the Non-Iterative Method can more easily be implemented in the data system of a wind tunnel as the load prediction of a given load component is completely independent of the load prediction of other load components. In addition, it was observed at the Ames Balance Calibration Laboratory that the load prediction accuracy of the Non-Iterative Method is as good as the load prediction accuracy of the Iterative Method as long as the chosen regression models of the balance calibration data do not have "hidden" linear or massive near-linear dependencies (a metric called the Variance Inflation Factor may be used to detect linear dependencies in a regression model). Therefore, it was decided to study a possible implementation of the Non-Iterative Method in the data system of the NASA Ames Unitary Plan Wind Tunnel in order to reduce the complexity of the load prediction process for balances that need either a temperature difference or a bellows pressure as input.

A regression model definition for a balance load is presented in the next section of the paper that supports both the use of output differences and state variables as independent variables. Afterwards, basic elements of the global regression analysis process are summarized that may be used to obtain the coefficients of the regression model of a load component. Finally, data from the machine calibration of a six-component force balance is used to illustrate application and load prediction accuracy of the Non-Iterative Method.

\section{Regression Model Definition}

It is assumed that a balance load component is a function of (i) the electrical outputs of the balance gages and (ii) state variables that influence the load prediction. It is also assumed that the balance has a total of " $n$ " load components that are represented by the symbols $F_{1}, F_{2}, \ldots, F_{n}$. They can be described as the components of a vector in an $n$-dimensional load space. Then, we get:

$$
[\mathbf{F}]_{n \times 1}=\left[\begin{array}{c}
F_{1} \\
\vdots \\
F_{i} \\
\vdots \\
F_{n}
\end{array}\right]
$$

The number of load components of a strain-gage balance cannot exceed six as a maximum of three forces and three moments can act at its balance moment center in 3-dimensional space. In addition, the maximum number of linearly independent electrical outputs of a strain-gage balance must always match the maximum number of applied load components of the balance if it is assumed that all state variables 
remain constant (see also the discussion of this topic in Ref. [5]). These two conclusions can be summarized as follows:

$$
n=\text { number of load components }=\text { number of gage output measurements } \leq 6
$$

The "load state" of a balance in the "load space" can uniquely be specified by the combination of loads that act on the balance at a given point in time. These loads are predicted during a wind tunnel test by using a set of "alternate" variables that also uniquely describe the "load state" of the balance. The electrical outputs of the balance gages are traditionally used for this purpose. Electrical outputs are often reported as absolute voltage measurements (raw outputs). This output format choice, however, makes the resulting regression model of a load component difficult to use during a wind tunnel test as (i) absolute voltage measurements frequently experience small instrumentation-induced shifts and (ii) significant instrumentation hardware differences often exist between wind tunnel and balance calibration laboratory. Alternatively, output differences relative to the natural zeros of the balance gages may be used to describe the "load state" of a balance in order to avoid these two problems. They may be defined as follows:

$$
\text { output difference of gage } j \Longrightarrow D_{j}=r F_{j}-N_{j} ; 1 \leq j \leq n
$$

In general, the use of output differences for the regression analysis of balance calibration data has three major benefits: (i) improved control of instrumentation-induced output shifts as long as raw outputs and natural zeros of the gages are measured by using the same instrumentation, (ii) better mathematical description of balance data symmetries and asymmetries near zero absolute load, and (iii) the use of the intercept in the regression model becomes optional as it is a least squares approximation of zero absolute load.

State variables need to be included in the list of alternate variables if they influence the load prediction. The alternate variables can be described in vector format by using the symbols $D_{1}, \ldots, D_{j}, \ldots, D_{m}$ assuming that (i) a total number of $m$ output differences and state variables are needed for the description of the "load state" of the balance and (ii) the symbol $D_{j}$ is also used for a state variable. Then, we get ...

$$
[\mathbf{D}]_{m \times 1}=\left[\begin{array}{c}
D_{1} \\
\vdots \\
D_{j} \\
\vdots \\
D_{m}
\end{array}\right]
$$

where the total number of "alternate" variables must always be greater than or equal to the number of load components. This requirement can be summarized as follows:

$$
m=n+\text { number of state variables }
$$

It is useful to construct an example so that the components of vectors $\mathbf{F}$ and $\mathbf{D}$ can be illustrated. It is assumed that the loads of a six-component balance are given in force balance format and that (i) its electrical outputs, (ii) natural zeros, and (iii) the difference between the balance temperature and a reference temperature are needed for the description of its "load state." Then, vectors $\mathbf{F}$ and $\mathbf{D}$ are defined as follows:

$$
\begin{aligned}
\text { load vector } \Longrightarrow[\mathbf{F}]_{6 \times 1}=\left[\begin{array}{c}
F_{1} \\
F_{2} \\
F_{3} \\
F_{4} \\
F_{5} \\
F_{6}
\end{array}\right]=\left[\begin{array}{c}
N 1 \\
N 2 \\
S 1 \\
S 2 \\
R M \\
A F
\end{array}\right] \\
\text { alternate load state description } \Longrightarrow[\mathbf{D}]_{7 \times 1}=\left[\begin{array}{c}
D_{1} \\
D_{2} \\
D_{3} \\
D_{4} \\
D_{5} \\
D_{6} \\
D_{7}
\end{array}\right]=\left[\begin{array}{c}
r N 2-N_{1} \\
r S 1-N_{3} \\
r S 2-N_{4} \\
r R M-N_{5} \\
r A F-N_{6} \\
T-T_{\circ}
\end{array}\right]
\end{aligned}
$$


Now, a regression model needs to be obtained from some balance calibration data in order to predict a load component $F_{i}$ of the balance from the set of alternate variables $D_{1}, D_{2}, \ldots, D_{m}$. It is assumed that the unknown functional relationship between the load component and the set of alternate variables could potentially be described by a second order Taylor series including additional cubic terms. This assumption results in four term groups that may be used to define the regression model of the balance calibration data. The Taylor series is developed in the neighborhood of zero absolute load. Ideally, each "alternate" variable should be described as a difference relative to the value that the variable would have at zero absolute load. Therefore, for example, the electrical outputs of the strain-gages should be computed as differences relative to the natural zeros of the balance (see also Eq. (3)). Similarly, the balance temperature needs to be expressed as a temperature difference relative to the reference temperature that the balance had when the natural zeros were recorded.

It is widely known that balances of Able/Task design have bi-directional outputs. This characteristic requires the use of absolute value terms of the loads in regression models of its outputs if the Iterative Method is used for the data analysis (see Ref. [2] and [6] for more detail). Each output can be approximated by the product of a constant, i.e., its primary gage sensitivity, and the related primary load component. In other words, an output is approximately proportional to the related load component. Therefore, absolute value terms of the outputs also need to be included in a regression model of a load if (i) a balance has bi-directional outputs and (ii) the Non-Iterative Method is used for the load prediction.

A total of six different term groups with absolute value terms are defined in the literature for use with bi-directional balance data (see, e.g., Ref. [2], Eq. (3.1.3)). However, it can rigorously be proven that two of the six absolute value term groups, i.e., $D_{j} \cdot\left|D_{k}\right|$ and $\left|D_{j}\right| \cdot D_{k}$ are related. The terms of these two groups can be expressed by all possible combinations of term group $D_{j} \cdot\left|D_{k}\right|$ without losing a single term if the limits of the related double sum are modified (a rigorous mathematical proof of this assertion can be found in the appendix of the paper). Therefore, five instead of six term groups may be used with absolute value terms for the regression model of a balance with bi-directional characteristics. Those five groups consist of one first order term group, three second order term groups, and one third order term group.

It is convenient to summarize the definition of the nine possible regression model term groups in table format. Table 1 below shows the term definitions of the term groups including term index ranges. The terms are displayed in Table 1 according to their order of magnitude (first order terms before second and third order terms). This order matches the term order that the Iterative Method uses for the regression model of an output (see Ref. [2], Eq. (3.1.3)). The index ranges are a function of (i) the total number of variables that are needed to describe the load state of the balance and (ii) index constraints that are related to the term definition itself.

Table 1: Regression model term group choices for the regression model a balance load component.

\begin{tabular}{|c|c|c|}
\hline$\nu \equiv$ Term Group Index & Term Definition & Index Range \& Constraints \\
\hline \hline 1 & $D_{j}$ & $1 \leq j \leq m$ \\
\hline 2 & $\left|D_{j}\right|$ & $1 \leq j \leq m$ \\
\hline 3 & $D_{j}^{2}$ & $1 \leq j \leq m$ \\
\hline 4 & $D_{j} \cdot\left|D_{j}\right|$ & $1 \leq j \leq m$ \\
\hline 5 & $D_{j} \cdot D_{k}$ & $1 \leq j \leq m$ and $1 \leq k \leq m$ where $j \neq k$ \\
\hline 6 & $\left|D_{j} \cdot D_{k}\right|$ & $1 \leq j \leq m$ and $1 \leq k \leq m$ where $j \neq k$ \\
\hline 7 & $D_{j} \cdot\left|D_{k}\right|$ & $1 \leq j \leq m$ and $1 \leq k \leq m$ where $j \neq k$ \\
\hline 8 & $D_{j}^{3}$ & $1 \leq j \leq m$ \\
\hline 9 & $\left|D_{j}^{3}\right|$ & $1 \leq j \leq m$ \\
\hline
\end{tabular}

${ }^{\dagger}$ Terms of groups $2,4,6,7,9$ are only to be used with data of balances that have gages with bi-directional characteristics.

The Non-Iterative Method fits each load component independent of all other load components. Therefore, it is sufficient to focus on a single load component during the discussion of the regression model definition. In addition, it is helpful to define a hypothetical upper bound of the regression model of a load 
component. This upper bound can be obtained after combining all possible terms that the term definitions listed in Table 1 above allow. An intercept term may also be included in the regression model definition. Then, the following upper bound of the regression model of load component $F_{i}$ is obtained where $a_{i, 0}$ is the

\section{Regression Model of a Balance Load Component}

$$
F_{i}=a_{i, 0}+\sum_{\nu=1}^{9} S_{\nu}=a_{i, 0}+\underbrace{a_{i, 1} \cdot D_{1}+a_{i, 2} \cdot D_{2}+\ldots+a_{i, \lambda} \cdot\left|D_{m}^{3}\right|}_{S_{1}+S_{2}+\ldots+S_{9}}
$$

intercept term (constant) and $S_{\nu}$ are either single or double sums that represent all possible terms for each one of the nine term groups of Table 1. Table 2 below lists the single/double sum $S_{\nu}$ and the related regression coefficient index range for each one of the nine term groups ( $\nu \equiv$ term group index).

Table 2: Summation definition and coefficient index ranges of regression model term groups.

\begin{tabular}{|c|c|c|c|}
\hline $\begin{array}{c}\nu \\
\text { (group index) }\end{array}$ & $\begin{array}{c}S_{\nu} \\
\text { (definition of summation) }\end{array}$ & $\begin{array}{c}\ldots \leq \xi \leq \ldots \\
\text { (coefficient index range) }\end{array}$ & $\begin{array}{c}\mu_{\nu} \\
\text { (number of terms) }\end{array}$ \\
\hline \hline 1 & $\sum_{j=1}^{m} a_{i, \xi} \cdot D_{j}$ & $1 \leq \xi \leq \mu_{1}$ & $m$ \\
\hline 2 & $\sum_{j=1}^{m} a_{i, \xi} \cdot\left|D_{j}\right|$ & $1+\mu_{1} \leq \xi \leq \sum_{\nu=1}^{2} \mu_{\nu}$ & $m$ \\
\hline 3 & $\sum_{j=1}^{m} a_{i, \xi} \cdot D_{j}^{2}$ & $1+\sum_{\nu=1}^{2} \mu_{\nu} \leq \xi \leq \sum_{\nu=1}^{3} \mu_{\nu}$ & $m$ \\
\hline 4 & $\sum_{j=1}^{m} a_{i, \xi} \cdot D_{j} \cdot\left|D_{j}\right|$ & $1+\sum_{\nu=1}^{3} \mu_{\nu} \leq \xi \leq \sum_{\nu=1}^{4} \mu_{\nu}$ & $m$ \\
\hline 5 & $\sum_{j=1}^{m-1} \sum_{k=j+1}^{m} a_{i, \xi} \cdot D_{j} \cdot D_{k}$ & $1+\sum_{\nu=1}^{4} \mu_{\nu} \leq \xi \leq \sum_{\nu=1}^{5} \mu_{\nu}$ & $\frac{m \cdot(m-1)}{2}$ \\
\hline 6 & $\sum_{j=1}^{m-1} \sum_{k=j+1}^{m} a_{i, \xi} \cdot\left|D_{j} \cdot D_{k}\right|$ & $1+\sum_{\nu=1}^{5} \mu_{\nu} \leq \xi \leq \sum_{\nu=1}^{6} \mu_{\nu}$ & $\frac{m \cdot(m-1)}{2}$ \\
\hline $7^{\dagger}$ & $\sum_{j=1}^{m} \sum_{k=1}^{m} a_{i, \xi} \cdot D_{j} \cdot\left|D_{k}\right|$ & $1+\sum_{\nu=1}^{6} \mu_{\nu} \leq \xi \leq \sum_{\nu=1}^{7} \mu_{\nu}$ & $m \cdot(m-1)$ \\
\hline$\sum_{k \neq j}^{m} a_{i, \xi} \cdot D_{j}^{3}$ & $1+\sum_{\nu=1}^{7} \mu_{\nu} \leq \xi \leq \sum_{\nu=1}^{8} \mu_{\nu}$ & $m$ \\
\hline$\sum_{j=1}^{m} a_{i, \xi} \cdot\left|D_{j}^{3}\right|$ & $1+\sum_{\nu=1}^{8} \mu_{\nu} \leq \xi \leq \sum_{\nu=1}^{9} \mu_{\nu}$ & $m$ \\
\hline & $\sum_{j=1}^{m}$ & $m$ & $m$ \\
\hline
\end{tabular}

${ }^{\dagger}$ Group 7 combines groups with terms $D_{j} \cdot\left|D_{k}\right|$ and $\left|D_{j}\right| \cdot D_{k}$ that are defined in Ref. [2], Eq. (3.1.3).

The values of variable $\mu_{\nu}$ of the last column of Table 2 above are the number of possible terms for each term group. It is interesting to compare the total number of possible terms of the proposed upper bound of the regression model of a load component with the number of terms that a corresponding regression model of 
an output would have if an analyst chooses to use the Iterative Method instead of the Non-Iterative Method for the balance load prediction. The total number of terms of the upper bound of the regression model of a balance load component is simply given by one plus the sum of all values that are listed in the last column of Table 2 above assuming the intercept term is included in the regression model. Then, we get:

\section{Maximum Number of Regression Model Terms}

$$
\lambda=1+\sum_{\nu=1}^{9} \mu_{\nu}=1+6 \cdot m+2 \cdot m \cdot(m-1)
$$

Now, let us assume for simplicity that a load component of a six-component force balance is predicted by exclusively using its six gage outputs (no state variables are used). Then, we get the following value for the total number of terms of the upper bound of the regression model of the load component:

$$
m=6 \quad \Longrightarrow \quad \lambda=1+36+60=97
$$

The value of 97 for the maximum number of terms of the regression model of a load matches the number of terms of the regression model of an output that the Iterative Method uses for a six-component balance. This result is expected because the individual terms of the term groups defined in Ref. [2] for the Iterative Method and in Table 1 for the Non-Iterative Method match keeping in mind that the terms of the "missing" group $\left|D_{j}\right| \cdot D_{k}$ are contained in group $D_{j} \cdot\left|D_{k}\right|$ (see also the proof given in the appendix of the paper).

Alternatively, let us assume that a load component of a six-component force balance is to be predicted by using (i) its six independent electrical outputs and (ii) the balance temperature difference relative to a reference temperature. Then, we get the following value for the total number of terms of the upper bound:

$$
m=7 \quad \Longrightarrow \quad \lambda=1+42+84=127
$$

It is important to remind the reader that Eq. (7) above simply describes all possible terms that the nine term groups can generate. A real-world calibration data set of a strain-gage balance will only support a subset of those terms. This conclusion is discussed in more detail in the next section of the paper.

\section{Global Regression Analysis}

The upper bound of the regression model of a balance load component was defined in Eq. (7) above. Real-world balance calibration data will often only allow for the use of a much smaller subset of all possible terms. This subset must be selected such that (i) the calibration data itself supports the chosen terms and (ii) no unwanted linear or near-linear dependencies exist between terms. In addition, it is recommended that the regression model be "hierarchical", i.e., that the model has no missing lower order terms (for more detail see discussion in Ref. [7], p. 204). Then, a robust and reliable regression model of a load component can be obtained that will not suffer from load prediction problems during use in the wind tunnel or will result in a singular solution of the global regression analysis problem of the calibration data.

The individual term selection may be made by using, for example, the Variance Inflation Factor for the detection of unwanted linear or near-linear dependencies between the regression model terms (see Ref. [7], p. 110). Alternatively, experienced analysts may use their own subject-matter knowledge during the regression model term selection. Afterwards, the global regression analysis of the calibration data can be performed so that the coefficients of the chosen regression model terms become known (see, e.g., Ref. [7], pp. $63-75$, for a description of the global regression analysis process).

Basic steps of the calculation of the regression coefficients of a load component using the global regression analysis process can be summarized in a few steps. Again, it is assumed that a subset of " $\delta$ " supported terms of Eq. (7) was found that leads to a non-singular solution during the regression analysis of the balance calibration data. Then the vector containing the dependent variables of the regression analysis problem, i.e., 
the calibration loads of load component $F_{i}$, needs to be defined. It can be written as follows assuming that the balance calibration data has a total number of " $p$ " data points:

$$
[\mathbf{b}]_{p \times 1}=\left[\begin{array}{c}
F_{i}(1) \\
F_{i}(2) \\
\vdots \\
F_{i}(p)
\end{array}\right]
$$

In the next step, a rectangular matrix containing the values of the regression model terms for each data point has to be assembled. This matrix has " $p$ " rows and " $\delta$ " columns. Let us assume, for example, that the intercept, linear terms, and a few other terms were chosen for the regression model. Then, the matrix can be described as follows:

$$
[\mathbf{A}]_{p \times \delta}=\underbrace{\left[\begin{array}{ccccc}
1 & D_{1}(1) & D_{2}(1) & D_{3}(1) & \cdots \\
1 & D_{1}(2) & D_{2}(2) & D_{3}(2) & \cdots \\
\vdots & \vdots & \vdots & \vdots & \vdots \\
1 & D_{1}(p) & D_{2}(p) & D_{3}(p) & \cdots
\end{array}\right]}_{\delta=\text { number of columns (regression model terms }) \leq \lambda}
$$

It is required that the column vectors of matrix $\mathbf{A}$ are linearly independent or have only moderate nearlinear dependencies. Only in that case a robust and reliable non-singular, i.e., unique, solution of the global regression analysis problem can be found. Finally, the unknown regression coefficients of the " $\delta$ " terms can be assembled in vector format. Then, we get:

$$
[\mathbf{x}]_{\delta \times 1}=\left[\begin{array}{c}
a_{i, 0} \\
a_{i, 1} \\
a_{i, 2} \\
a_{i, 3} \\
\vdots
\end{array}\right]
$$

At this point, the global least squares problem associated with the fit of the load component as a function of electrical outputs and state variables can be described as an overdetermined linear system of equations. We get the following matrix equation:

\section{Global Least Squares Problem}

$$
[\mathbf{A}]_{p \times \delta} \cdot[\mathbf{x}]_{\delta \times 1}=[\mathbf{b}]_{p \times 1}
$$

The elements of rectangular matrix $\mathbf{A}$ and column vector $\mathbf{b}$ are known because they were obtained from the loads, outputs, and state variables that were recorded during the balance calibration. It is shown in the literature that the unknown regression coefficients of the global least squares problem defined in Eq. (11) above are the solution of the related Normal Equations (see, e.g., Ref. [7] for more details). In our case, these equations are defined as follows:

\section{Normal Equations of Global Least Squares Problem}

$$
\left[\mathbf{A}^{\mathbf{T}} \mathbf{A}\right]_{\delta \times \delta} \cdot[\mathbf{x}]_{\delta \times 1}=\left[\mathbf{A}^{\mathbf{T}}\right]_{\delta \times p} \cdot[\mathbf{b}]_{p \times 1}
$$


The solution of the Normal Equations can be computed by multiplying both sides of Eq. (12) with the inverse of square matrix $\mathbf{A}^{\mathbf{T}} \mathbf{A}$. It equals the solution of the global regression analysis problem. We get:

\section{Regression Coefficients $\equiv$ Solution of Global Least Squares Problem}

$$
[\mathbf{x}]_{\delta \times 1}=\underbrace{\left[\left(\mathbf{A}^{\mathbf{T}} \mathbf{A}\right)^{-\mathbf{1}}\right]_{\delta \times \delta} \cdot\left[\mathbf{A}^{\mathbf{T}}\right]_{\delta \times p}}_{\text {independent of } F_{i}(1), \ldots, F_{i}(p)} \cdot[\mathbf{b}]_{p \times 1}
$$

The given solution of the regression coefficients above is valid for load component $F_{i}$ as the related calibration load values $F_{i}(1), F_{i}(2), \ldots, F_{i}(p)$ are contained in vector $\mathbf{b}$. The regression model of $F_{i}$ itself is "hidden" within matrix A. Its coefficients were constructed by using (i) the supported regression model terms, (ii) output differences of the balance gages relative to the natural zeros, and, if applicable, (iii) state variable values of the calibration data as input.

The final regression model of a balance calibration data set is highly dependent on the calibration load schedule. Therefore, the set of supported terms of a load component can vary from balance to balance and also from calibration data set to calibration data set. Consequently, the authors suggest to always use the upper bound of the regression model as a fixed output format for the reporting of the coefficients of a load component even though it means that a large subset of coefficients will be zero. This approach makes it easier to implement a universally applicable matrix format in the data system of a wind tunnel that is independent of the chosen balance and calibration load schedule. More details can be found in the next section.

\section{Balance Load Prediction Equations}

Equation (13) above specifies coefficients of the regression model of a single load component $F_{i}$ of the balance. Coefficients of other load components have to be obtained by updating the contents of (i) matrix A that has the regression model terms derived from all electrical outputs of the $n$-component balance and (ii) column vector $\mathbf{b}$ that has the calibration loads $F_{i}(1), \ldots, F_{i}(p)$ of the chosen load component. Afterwards, coefficients of all load components can be stored in a rectangular matrix so that a single matrix equation may be used for the simultaneous calculation of all load components during a wind tunnel test.

The rectangular matrix needed for the calculation of all load components is obtained by first "mapping" column vector $\mathbf{x}$ with the coefficients of a single load component from the supported " $\delta$ " terms to the maximum number of " $\lambda$ " terms. The mapping is done without changing the result of the regression analysis of the load as coefficients of unused regression model terms of Eq. (7) are explicitly set to "0". Then, the transformed solution vector of the coefficients of the regression model of a single load component with index $i$ can be expressed as follows:

$$
\{\text { supported terms }(\delta) \Longrightarrow \text { all terms }(\lambda)\} \quad \Longrightarrow \quad\left\{[\mathbf{x}]_{\delta \times 1} \Longrightarrow[\mathbf{y}]_{\lambda \times 1}\right\}
$$

The number and type of supported terms of the total number of the $n$ final regression models of the loads of the balance may or may not be the same depending on an analyst's term selections. However, the transformed solution vectors $\mathbf{x}$ of the coefficients of each single load can be combined in a rectangular matrix with a total of " $\lambda$ " rows and " $n$ " columns after coefficients of each load were mapped one by one from the supported terms to the maximum number of terms. This conclusion can be summarized as follows:

$$
\{\text { single load }(1) \Longrightarrow \text { all loads }(n)\} \Longrightarrow\left\{[\mathbf{y}]_{\lambda \times 1} \Longrightarrow[\mathbf{z}]_{\lambda \times n}\right\}
$$

Similarly, it is necessary to transform the columns of matrix A from the number of " $\delta$ " supported terms to the maximum number of " $\lambda$ " terms. This time, however, matrix A consists of a single row as only data from a single data point is needed for the prediction of the balance loads for a given set of output differences and other state variables that are recorded during a wind tunnel test. Then, we get:

$$
\{\text { supported terms }(\delta) \Longrightarrow \text { all terms }(\lambda)\} \quad \Longrightarrow \quad\left\{[\mathbf{A}]_{1 \times \delta} \Longrightarrow[\mathbf{B}]_{1 \times \lambda}\right\}
$$


By inspection, the matrix equation describing the calculation of the loads of an $n$-component balance during a wind tunnel test can be expressed as follows:

$$
\left[\mathbf{F}^{\mathbf{T}}\right]_{1 \times n}=\left[\begin{array}{llll}
F_{1} & F_{2} & \ldots & F_{n}
\end{array}\right]=[\mathbf{B}]_{1 \times \lambda} \cdot[\mathbf{z}]_{\lambda \times n}
$$

Finally, after taking the transpose of both sides of the Eq. (15) and knowing that $(\mathbf{X Y})^{\mathbf{T}}=\mathbf{Y}^{\mathbf{T}} \mathbf{X}^{\mathbf{T}}$ (from Ref. [8], p. 334), we get the final set of balance load prediction equations for the Non-Iterative Method:

\section{Non-Iterative Balance Load Prediction Equations}

$$
[\mathbf{F}]_{n \times 1}=\left[\begin{array}{c}
F_{1} \\
\vdots \\
F_{i} \\
\vdots \\
F_{n}
\end{array}\right]=\underbrace{\left[\mathbf{z}^{\mathbf{T}}\right]_{n \times \lambda}}_{\text {coefficients }} \cdot \underbrace{\left[\mathbf{B}^{\mathbf{T}}\right]_{\lambda \times 1}}_{\text {model terms }}
$$

where

$$
\begin{aligned}
{\left[\mathbf{z}^{\mathbf{T}}\right]_{n \times \lambda} } & =\left[\begin{array}{ccccc}
a_{1,0} & a_{1,1} & a_{1,2} & a_{1,3} & \ldots \\
\vdots & \vdots & \vdots & \vdots & \vdots \\
a_{i, 0} & a_{i, 1} & a_{i, 2} & a_{i, 3} & \ldots \\
\vdots & \vdots & \vdots & \vdots & \vdots \\
a_{n, 0} & a_{n, 1} & a_{n, 2} & a_{n, 3} & \ldots
\end{array}\right] \\
& {\left[\mathbf{B}^{\mathbf{T}}\right]_{\lambda \times 1}=\left[\begin{array}{c}
1 \\
D_{1} \\
D_{2} \\
D_{3} \\
\vdots
\end{array}\right] }
\end{aligned}
$$

Data from a machine calibration of a six-component force balance is used in the next section to illustrate typical results if the Non-Iterative Method is used for the balance load prediction.

\section{Discussion of Example}

Data from a 2018 machine calibration of a force balance is used in this section to illustrate the application of Non-Iterative Method to a calibration data set. The chosen balance is called the NASA MK34A. It was manufactured by the Able/Task Corporation. Figure 1 below shows the general lay-out of the balance.

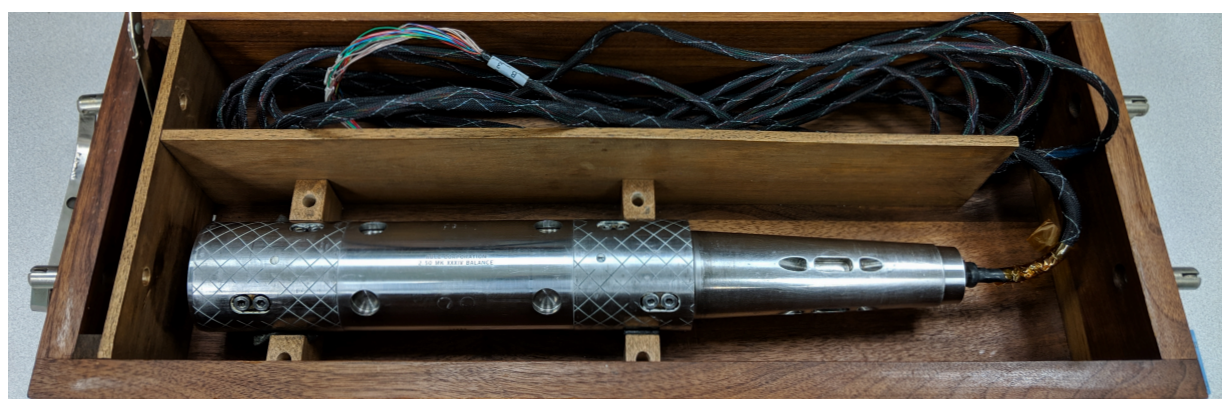

Fig. 1 NASA's 2.5 inch diameter MK34A six-component force balance. 
The MK34A is a six-component force balance that measures five forces and one moment $(N 1, N 2, S 1$, $S 2, A F, R M)$. It has a diameter of 2.5 inches and a total length of 17.31 inches. Table 3 shows the capacity of each load component of the balance.

Table 3: Load capacities of NASA's 2.5 inch MK34A force balance.

\begin{tabular}{|c|c|c|c|c|c|}
\hline$N 1, \mathrm{lbs}$ & $N 2, \mathrm{lbs}$ & $S 1, \mathrm{lbs}$ & $S 2, \mathrm{lbs}$ & $A F, \mathrm{lbs}$ & $R M$, in-lbs \\
\hline \hline 3500 & 3500 & 1800 & 1800 & 400 & 5000 \\
\hline
\end{tabular}

The balance was calibrated using CALSPAN San Diego's Automatic Balance Calibration System (ABCS). The calibration load schedule consisted of a total of 2055 data points that were distributed across 16 load series. Up to five load components were applied simultaneously during the calibration.

NASA's BALFIT software package was used for the analysis of the machine calibration data of the MK34A balance (see Ref. [9] for a description of the software; it supports the application of both the Iterative and Non-Iterative Method to strain-gage balance data). The MK34A is a typical Able/Task balance design with known bi-directional normal and side force gage output characteristics. Therefore, absolute value terms were included in the regression models of the six load components. A tare load iteration was applied during the analysis so that the weight of the metric part of the balance and all attached calibration hardware pieces would be included in the analysis. The temperature of the balance was not varied during the calibration. Therefore, each load component could only be modeled as a function of the six measured output differences after the natural zero of each gage was subtracted from the corresponding absolute voltage measurement. In addition, regression model term reduction was performed so that "overfitting" of the tare corrected calibration data would be avoided.

Table 4 below shows the standard deviations of the load residuals of the calibration data, i.e., the standard deviations of the difference between tare corrected and fitted loads, that were obtained for each load component (the number of regression model terms of each load component is listed in brackets). The standard deviation is expressed as a percentage of the load capacity.

Table 4: Non-Iterative Method $\Longrightarrow$ Standard deviation of calibration load residuals (the number of regression model terms of each component is listed in brackets).

\begin{tabular}{|c|c|c|c|c|c|}
\hline$N 1$ & $N 2$ & $S 1$ & $S 2$ & $A F$ & $R M$ \\
\hline \hline $0.0577 \%$ & $0.0570 \%$ & $0.1556 \%$ & $0.1748 \%$ & $0.1123 \%$ & $0.0594 \%$ \\
$(33$ terms $)$ & $(34$ terms $)$ & $(32$ terms $)$ & $(30$ terms $)$ & $(39$ terms $)$ & $(35$ terms $)$ \\
\hline
\end{tabular}

It was also decided to analyze the original calibration data of the MK34A balance by using the alternate Iterative Method so that a load prediction accuracy comparison could directly be performed. The same analysis constraints were used during the application of the Iterative Method to the calibration data (inclusion of absolute value terms, tare load iteration, regression model term reduction). Table 5 below shows the standard deviations of the load residuals of the calibration data after the Iterative Method was applied (the number of chosen regression model terms of each gage output is shown in brackets).

Table 5: Iterative Method $\Longrightarrow$ Standard deviation of calibration load residuals (the number of regression model terms of each component is listed in brackets).

\begin{tabular}{|c|c|c|c|c|c|}
\hline$N 1$ & $N 2$ & $S 1$ & $S 2$ & $A F$ & $R M$ \\
\hline \hline $0.0574 \%$ & $0.0580 \%$ & $0.1478 \%$ & $0.1722 \%$ & $0.1127 \%$ & $0.0596 \%$ \\
$(35$ terms $)$ & $(35$ terms $)$ & $(34$ terms $)$ & $(29$ terms $)$ & $(41$ terms $)$ & $(34$ terms $)$ \\
\hline
\end{tabular}

The standard deviations of the load residuals obtained for the Non-Iterative Method (Table 4) show excellent agreement with corresponding standard deviations for the Iterative Method (Table 5). Similar observations were made when data from a manual calibration of a six-component force balance and a manual calibration of a five-component semi-span balance were analyzed by using the same approach that 
was used for the MK34A balance. They confirm the authors' experience that the load prediction accuracy of the Non-Iterative Method is as good as the load prediction accuracy of the Iterative Method as long as (i) similar regression model term types are used for the analysis and (ii) the chosen regression models for the application of the two methods do not have linear or massive near-linear dependencies.

It is possible to take a "microscopic" look at the individual load residuals of a load component after either the Non-Iterative Method or the Iterative Method is applied to the calibration data. Figure 2a below, for example, shows the load residuals of the forward normal force component $(N 1)$ plotted versus the calibration data point index after the Non-Iterative Method was used for the analysis.

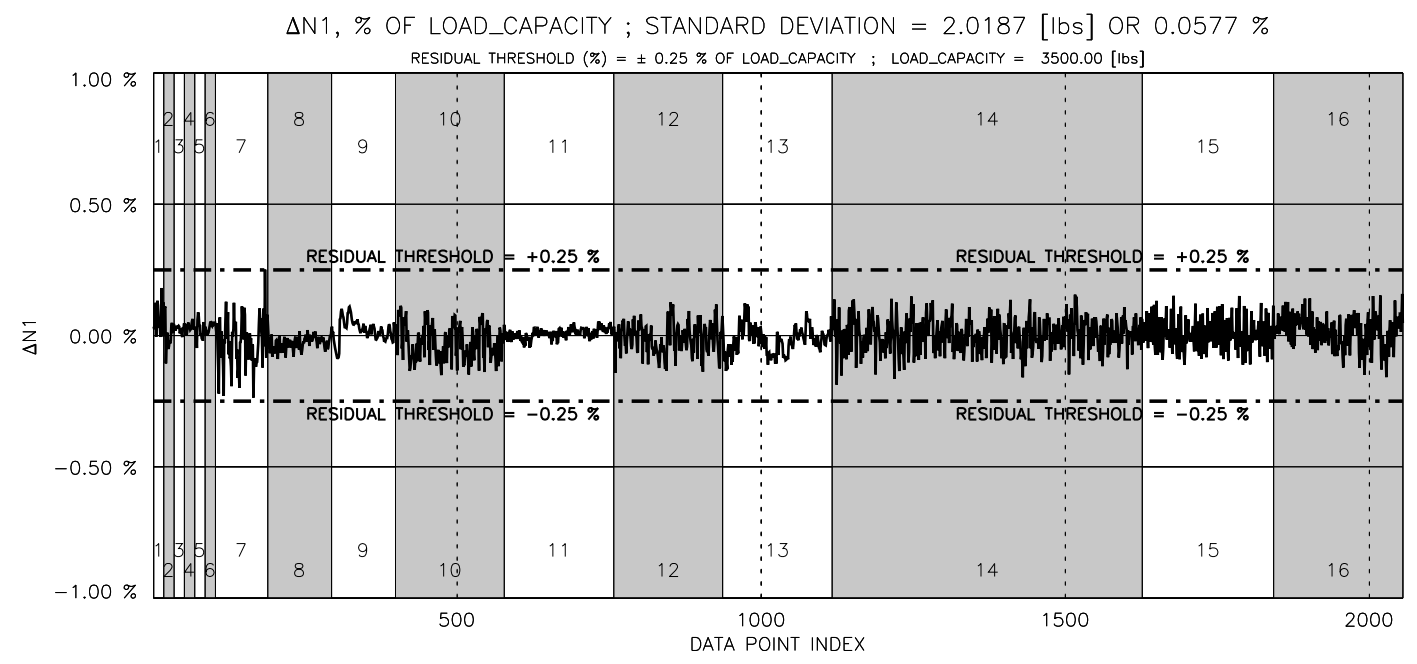

Fig. 2a Non-Iterative Method $\Longrightarrow$ Load residuals of the forward normal force of the MK34A.

Similarly, Fig. $2 \mathrm{~b}$ below shows the load residuals of the forward normal force component $(N 1)$ plotted versus the data point index after the Iterative Method was used for the analysis.

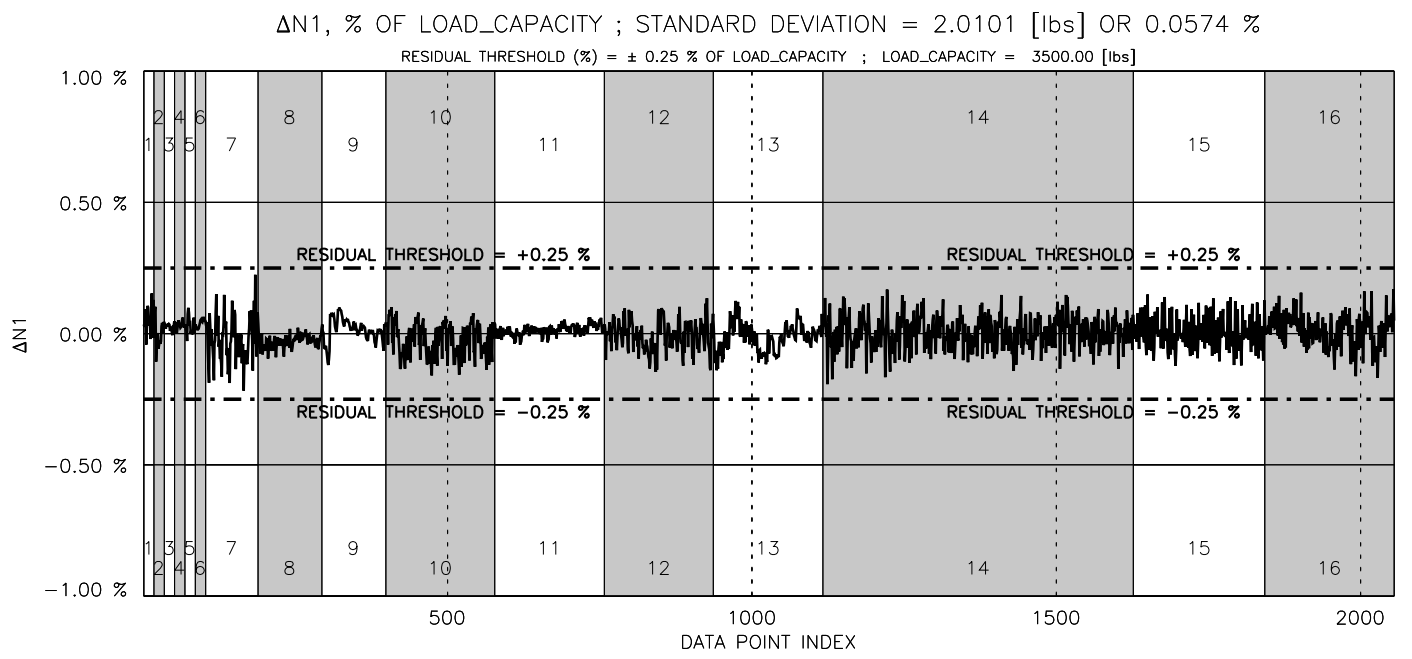

Fig. 2b Iterative Method $\Longrightarrow$ Load residuals of the forward normal force of the MK34A.

A qualitative comparison of the two plots above shows that even small features of the load residuals of individual load series are similar. The same observations were made for the other five load components. This conclusion explains why the standard deviations reported in Tables 4 and 5 are so close in magnitude. 
Basic characteristics of the regression models of the six load components can also be examined in more detail by a using a variety of objective metrics. The forward normal force is discussed as an example to illustrate typical results (results for the other five load components of the balance were similar). The Standard Error, $t$-Statistic, $p$-Value, and Variance Inflation Factor (VIF) of its 33 coefficients and regression model terms were computed (see, e.g., Ref. [7] for a description of these four metrics). Table 6 below shows the computed results for these four metrics for each term of the regression model of the forward normal force.

Table 6: Characteristics of the regression model of the forward normal force.

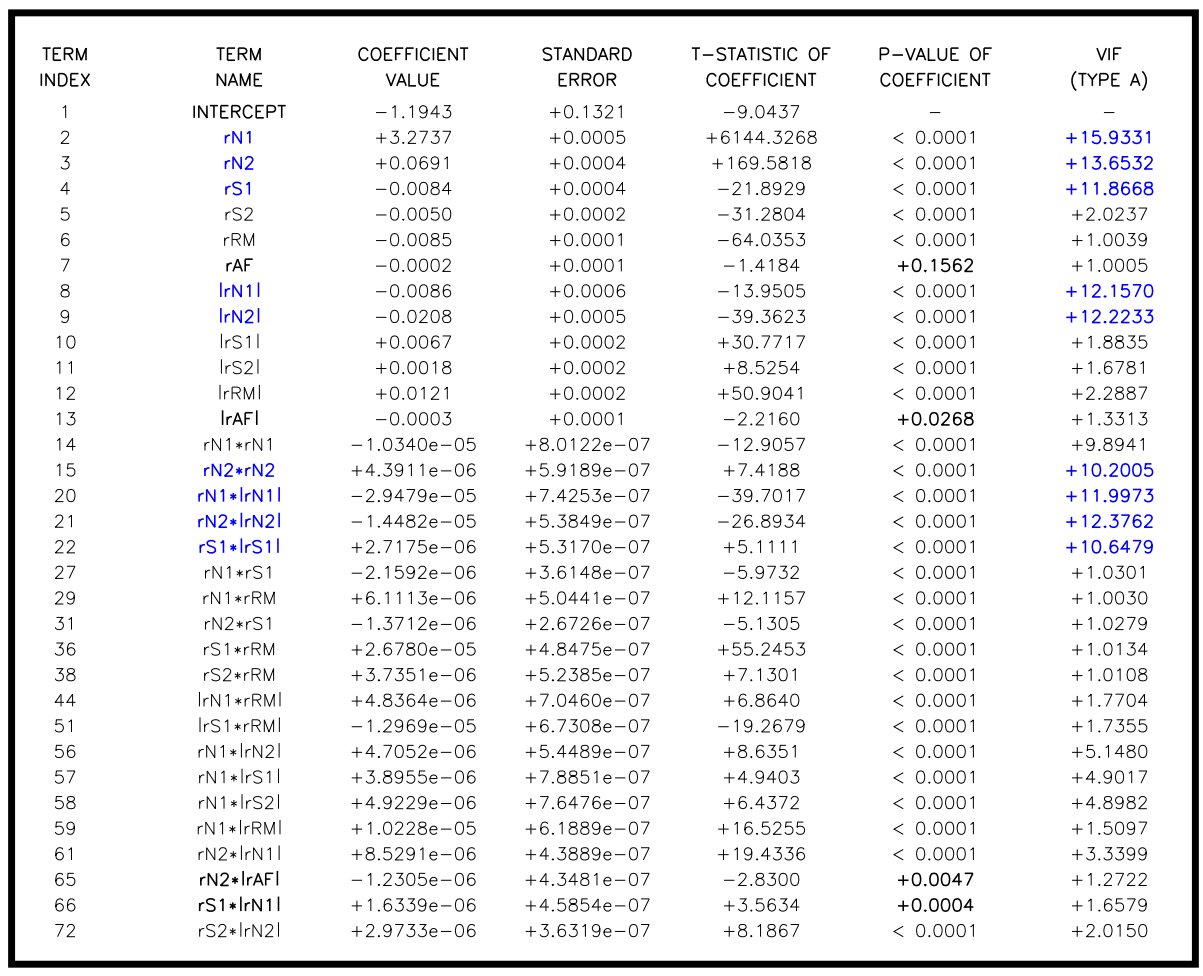

Several observations can be made after results of Table 6 above are examined in detail. For example, it can be seen that the coefficient of the forward normal force gage output $(r N 1)$ has by far the highest value of the t-Statistic (+6144). This result is expected as the forward normal force gage output of a force balance must, by design, explain most of the magnitude of the forward normal force that acts on the balance. In addition, the $p$-Value of 29 of the 33 coefficients is $<0.0001$. This result means that most coefficients chosen by BALFIT's regression model term reduction process are statistically significant. Only the two terms $r A F$ and $|r A F|$ appear to be "insignificant" in the regression model of the forward normal force as their $p$-Values are substantially greater that the threshold of 0.0001 (the $p$-Values of the two terms are 0.1562 and 0.0268 ). Finally, the maximum VIF of the selected regression model term combination is 15.9331 (the color blue marks VIFs that exceed the threshold of 10). This result indicates that only moderate near-linear dependencies exist in the regression model of the forward normal force.

\section{Summary}

A non-iterative load prediction process for wind tunnel strain-gage balances was developed that directly computes the loads of an $n$-component balance from (i) the difference between the electrical outputs of the gages and the related natural zeros and (ii) state variables (if applicable). The process uses a regression model of each balance load for the load prediction that is obtained from a global regression analysis of balance calibration data.

The terms of the regression model of a load are chosen from a total of nine term groups. Some term groups are derived from a Taylor series expansion of the functional relationship between the load and the electrical outputs and state variables. Term groups with absolute value terms are also included in the list of 
potential regression model terms so that data of balances with known bi-directional output characteristics can be described correctly. First, the coefficients of the regression model of each load component are computed by applying global regression analysis to the given balance calibration data. Afterwards, the coefficients are "mapped" to a rectangular matrix of fixed format so that the proposed Non-Iterative Method can easily be implemented in the data system of a wind tunnel facility.

Data from the machine calibration of a force balance was used to illustrate both application and performance of the proposed implementation of the Non-Iterative Method. Overall, it was observed for the given balance calibration data that the load prediction accuracy of the Non-Iterative Method is as good as the load prediction accuracy of the Iterative Method as long as (i) similar constraints are applied during the regression model selection and (ii) the chosen models of both methods do not have any linear or massive near-linear dependencies. However, the Non-Iterative Method has the advantage that it is more easily implemented and used in the data system of a wind tunnel.

\section{Acknowledgements}

The authors want to thank Bethany White and Bob Gisler of NASA and Tom Bridge of Jacobs Technology for their critical and constructive review of the manuscript. The work reported in this document was supported by the Wind Tunnel Division at NASA Ames Research Center under contract NNA16BD26C.

\section{References}

[1] Ulbrich, N., and Zimmermann, C., "Application of a Temperature-Dependent Load Prediction Method to a RUAG Six-Component Block-Type Balance," AIAA 2019-2294, paper presented at the 2019 AIAA SciTech Forum, San Diego, California, January 2019.

[2] AIAA/GTTC Internal Balance Technology Working Group, Recommended Practice, Calibration and Use of Internal Strain-Gage Balances with Application to Wind Tunnel Testing, AIAA R-091-2003, American Institute of Aeronautics and Astronautics, Reston, Virginia, 2003.

[3] Ulbrich, N., "Iterative Strain-Gage Balance Calibration Data Analysis for Extended Independent Variable Sets," AIAA 2011-0949, paper presented at the 49th AIAA Aerospace Sciences Meeting, Orlando, Florida, January 2011.

[4] Ulbrich, N., "Comparison of Iterative and Non-Iterative Strain-Gage Balance Load Calculation Methods," AIAA 2010-4202, paper presented at the 27th AIAA Aerodynamic Measurement Technology and Ground Testing Conference, Chicago, Illinois, June/July 2010.

[5] Ulbrich, N., "Assessment of the Uniqueness of Strain-Gage Balance Load Predictions," AIAA 20164157, paper presented at the 32nd AIAA Aerodynamic Measurement Technology and Ground Testing Conference, Washington, D.C., June 2016.

[6] Kammeyer, M. E., and Ulbrich, N., "The Use of Absolute-Value Terms in Regression Modeling of MultiPiece Force Balances," AIAA 2015-3382, paper presented at the 31st AIAA Aerodynamic Measurement Technology and Ground Testing Conference, Dallas, Texas, June 2015.

[7] Montgomery, D. C., Peck, E. A., and Vining, G. G., Introduction to Linear Regression Analysis, 4th ed., John Wiley \& Sons, Inc., New York, 2006; pp. 63-75, p. 110.

[8] Burden, R. L., and Faires, J. D., Numerical Analysis, 3rd edition, PWS-Kent Publishing Company, Boston, Massachusetts, 1985, p. 334.

[9] Ulbrich, N., and Volden, T., BALFIT - Software Tool for the Regression Analysis of Multivariate Data, User Guide (revised and corrected 6th edition), Jacobs Technology, prepared for NASA Ames Research Center under contract NNA16BD26C, May 2019.

[10] Rosen, K. H., Michaels, J. G., Gross, J. L., Grossman, J. W., Shier, D. R., Handbook of Discrete and Combinatorial Mathematics, CRC Press LLC, 2000, Section 3.5.2 (Elementary Transformation Rules for Sums), Fact 8 (Interchanging dependent indices of a double sum). 


\section{Appendix: Mathematical Proof of Term Group Simplification ${ }^{\dagger}$}

Different groups of regression model terms may be used to model strain-gage balance data so that balance loads may be predicted from electrical outputs during a wind tunnel test. A user of the Iterative Method, for example, constructs these term groups from the loads of the balance as this load prediction approach uses regression models of the electrical outputs for the definition of a load iteration scheme. Similarly, a user of the Non-Iterative Method constructs the term groups from the electrical outputs of the balance as this alternate load prediction approach directly generates regression models of the loads.

AIAA's Internal Balance Technology Working Group (IBTWG) recommended in 2003 a regression model term group combination for the Iterative Method that consists, superficially viewed, of ten term groups (see Ref. [2], Eq. (3.1.3)). However, it can rigorously be proven that two of the ten groups, i.e., $F_{i} \cdot\left|F_{j}\right|$ and $\left|F_{i}\right| \cdot F_{j}$, model the same characteristic in balance data. They can be combined in a single term group whose terms are defined as $F_{i} \cdot\left|F_{j}\right|$. This simplification is possible because the following equality is valid where $F_{i} \cdot\left|F_{j}\right|$ and $\left|F_{i}\right| \cdot F_{j}$ are regression model terms and $\alpha_{i, j}, \beta_{i, j}$, and $\gamma_{i, j}$ are regression coefficients:

$$
\sum_{i=1}^{n} \sum_{\substack{j=1 \\ j \neq i}}^{n} \alpha_{i, j} \cdot F_{i} \cdot\left|F_{j}\right|=\sum_{i=1}^{n-1} \sum_{j=i+1}^{n} \beta_{i, j} \cdot F_{i} \cdot\left|F_{j}\right|+\sum_{i=1}^{n-1} \sum_{j=i+1}^{n} \gamma_{i, j} \cdot\left|F_{i}\right| \cdot F_{j}
$$

The relationship above simply describes the fact that all combinations of variable $F_{i}$ with variable $\left|F_{j}\right|$ on both sides of Eq. (17) match even though the term order itself is not the same. In other words, it is always possible to identify a term $\alpha_{i, j} \cdot F_{i} \cdot\left|F_{j}\right|$ on the left-hand side of Eq. (17) that matches either a term $\beta_{i, j} \cdot F_{i} \cdot\left|F_{j}\right|$ or a term $\gamma_{i, j} \cdot\left|F_{i}\right| \cdot F_{j}$ on the right-hand side. The validity of Eq. (17) above means that $I B T W G$ 's regression model of an electrical output of a balance has two groups, i.e., $F_{i} \cdot\left|F_{j}\right|$ and $\left|F_{i}\right| \cdot F_{j}$, that are related to each other. They must either be used together or not at all in the regression model of an output if data from a balance with bi-directional outputs is processed. It must be mentioned at this point that known typographical errors exist in the four double sums that are defined in Ref. [2], Eq. (3.1.3). These typographical errors are responsible for an incorrect assignment of the upper limits of the related outer sums. These limits must be changed from the incorrect value of " $n$ " to the correct value of " $n-1$ ". The typographical errors were corrected in Eq. (17) above. The errors will also be corrected in an updated and extended version of Ref. [2] that is currently in preparation.

An analytical proof of Eq. (17) can be developed after omitting the regression coefficients themselves because it only needs to be demonstrated that the possible term combinations of $F_{i}$ and $\left|F_{j}\right|$ on both sides of Eq. (17) match. Consequently, we get the following alternate relationship that needs to be proven:

$$
\sum_{i=1}^{n} \sum_{\substack{j=1 \\ j \neq i}}^{n} F_{i} \cdot\left|F_{j}\right|=\sum_{i=1}^{n-1} \sum_{j=i+1}^{n} F_{i} \cdot\left|F_{j}\right|+\sum_{i=1}^{n-1} \sum_{j=i+1}^{n}\left|F_{i}\right| \cdot F_{j}
$$

The proof of Eq. (18) above can be done with the help of simple algebraic operations after the Kronecker delta is used to describe its left-hand side in a format that avoids the summation index constraint " $j \neq i$ ". By inspection, the following relationship is valid for the left-hand side of Eq. (18):

$$
\sum_{i=1}^{n} \sum_{\substack{j=1 \\ j \neq i}}^{n} F_{i} \cdot\left|F_{j}\right|=\sum_{i=1}^{n} \sum_{j=1}^{n}\left(1-\delta_{i j}\right) \cdot F_{i} \cdot\left|F_{j}\right|
$$

where

$$
\text { Kronecker delta } \Longrightarrow \delta_{i j}=\left\{\begin{array}{lll}
0 & \text { if } & i \neq j \\
1 & \text { if } & i=j
\end{array}\right.
$$

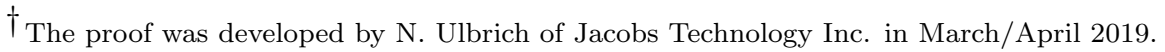


Then, after replacing the left-hand side of Eq. (18) with the right-hand side of Eq. (19a), we get for Eq. (18):

$$
\sum_{i=1}^{n} \sum_{j=1}^{n}\left(1-\delta_{i j}\right) \cdot F_{i} \cdot\left|F_{j}\right|=\sum_{i=1}^{n-1} \sum_{j=i+1}^{n} F_{i} \cdot\left|F_{j}\right|+\sum_{i=1}^{n-1} \sum_{j=i+1}^{n}\left|F_{i}\right| \cdot F_{j}
$$

The proof of Eqs. (18) and (20) is possible if the following "strategy" is applied: modify the left-hand side of Eq. (20) above until all terms on the right-hand side of Eq. (20) are obtained. The proof starts by first expanding the round brackets on the left-hand side of Eq. (20). Then, we get the following relationship

$$
L \equiv \sum_{i=1}^{n} \sum_{j=1}^{n}\left(1-\delta_{i j}\right) \cdot F_{i} \cdot\left|F_{j}\right|=\sum_{i=1}^{n} \sum_{j=1}^{n} F_{i} \cdot\left|F_{j}\right|-\sum_{i=1}^{n} \sum_{j=1}^{n} \delta_{i j} \cdot F_{i} \cdot\left|F_{j}\right|
$$

where symbol " $L$ " is used to abbreviate the left-hand side of Eq. (20). Now, the right-hand side of Eq. (21) above needs to be modified until the right-hand side of Eq. (20) is obtained in order to complete the proof. The first double sum on the right-hand side of Eq. (21) can be split into a double sum and a single sum after treating terms associated with the condition " $i=n$ " separately. Then, we get the following alternate version of the first double sum on the right-hand side of Eq. (21):

$$
\sum_{i=1}^{n} \sum_{j=1}^{n} F_{i} \cdot\left|F_{j}\right|=\sum_{i=1}^{n-1} \sum_{j=1}^{n} F_{i} \cdot\left|F_{j}\right|+\sum_{j=1}^{n} F_{n} \cdot\left|F_{j}\right|
$$

The inner sum of the double sum on the right-hand side of Eq. (22a) can be split into two parts. Then, we get the following alternate version of Eq. $(22 a)$ :

$$
\sum_{i=1}^{n} \sum_{j=1}^{n} F_{i} \cdot\left|F_{j}\right|=\sum_{i=1}^{n-1}\left[\sum_{j=1}^{i} F_{i} \cdot\left|F_{j}\right|+\sum_{j=i+1}^{n} F_{i} \cdot\left|F_{j}\right|\right]+\sum_{j=1}^{n} F_{n} \cdot\left|F_{j}\right|
$$

Now, after expanding the brackets on the right-hand side of Eq. (22b), we get:

$$
\sum_{i=1}^{n} \sum_{j=1}^{n} F_{i} \cdot\left|F_{j}\right|=\sum_{i=1}^{n-1} \sum_{j=1}^{i} F_{i} \cdot\left|F_{j}\right|+\sum_{i=1}^{n-1} \sum_{j=i+1}^{n} F_{i} \cdot\left|F_{j}\right|+\sum_{j=1}^{n} F_{n} \cdot\left|F_{j}\right|
$$

The index of the single sum on the right-hand side of Eq. (22c) can be switched from " $j$ " to " $i$ " without changing the result. Then, we get:

$$
\sum_{i=1}^{n} \sum_{j=1}^{n} F_{i} \cdot\left|F_{j}\right|=\sum_{i=1}^{n-1} \sum_{j=1}^{i} F_{i} \cdot\left|F_{j}\right|+\sum_{i=1}^{n-1} \sum_{j=i+1}^{n} F_{i} \cdot\left|F_{j}\right|+\sum_{i=1}^{n} F_{n} \cdot\left|F_{i}\right|
$$

In addition, we know from the Commutative Law that $F_{n} \cdot\left|F_{i}\right|$ equals $\left|F_{i}\right| \cdot F_{n}$. Then, we get:

$$
\sum_{i=1}^{n} \sum_{j=1}^{n} F_{i} \cdot\left|F_{j}\right|=\underbrace{\sum_{i=1}^{n-1} \sum_{j=1}^{i} F_{i} \cdot\left|F_{j}\right|}_{\text {First Double Sum }}+\underbrace{\sum_{i=1}^{n-1} \sum_{j=i+1}^{n} F_{i} \cdot\left|F_{j}\right|}_{\text {Second Double Sum }}+\sum_{i=1}^{n}\left|F_{i}\right| \cdot F_{n}
$$

The Commutative Law also allows us to switch the order of the addition of the two double sums on the right-hand side of Eq. (22e) above without changing the result. Then, we get:

$$
\sum_{i=1}^{n} \sum_{j=1}^{n} F_{i} \cdot\left|F_{j}\right|=\underbrace{\sum_{i=1}^{n-1} \sum_{j=i+1}^{n} F_{i} \cdot\left|F_{j}\right|}_{\text {Second Double Sum }}+\underbrace{\sum_{i=1}^{n-1} \sum_{j=1}^{i} F_{i} \cdot\left|F_{j}\right|}_{\text {First Double Sum }}+\sum_{i=1}^{n}\left|F_{i}\right| \cdot F_{n}
$$

American Institute of Aeronautics and Astronautics 
It remains to simplify the second double sum on the right-hand side of Eq. (21). This double sum can be substituted by a single sum after realizing that the summation term of the double sum only has a non-zero value if summation index " $i$ " equals summation index " $j$ ". Then, we get:

$$
\sum_{i=1}^{n} \sum_{j=1}^{n} \delta_{i j} \cdot F_{i} \cdot\left|F_{j}\right|=\sum_{i=1}^{n} F_{i} \cdot\left|F_{i}\right|
$$

We also know from the Commutative Law that $F_{i} \cdot\left|F_{i}\right|$ equals $\left|F_{i}\right| \cdot F_{i}$. Then, we get for Eq. (23a):

$$
\sum_{i=1}^{n} \sum_{j=1}^{n} \delta_{i j} \cdot F_{i} \cdot\left|F_{j}\right|=\sum_{i=1}^{n}\left|F_{i}\right| \cdot F_{i}
$$

In the next step, after replacing the right-hand side of Eq. (21) by the difference between the right-hand side of Eq. (22f) and the right-hand side of Eq. (23b), we get the following alternate version of Eq. (21):

$$
\Longrightarrow L=\underbrace{\sum_{i=1}^{n-1} \sum_{j=i+1}^{n} F_{i} \cdot\left|F_{j}\right|+\sum_{i=1}^{n-1} \sum_{j=1}^{i} F_{i} \cdot\left|F_{j}\right|+\sum_{i=1}^{n}\left|F_{i}\right| \cdot F_{n}}_{\text {Equation (22f) }}-\underbrace{\sum_{i=1}^{n}\left|F_{i}\right| \cdot F_{i}}_{\text {Equation (23b) }}
$$

The difference between the two single sums on the right-hand side of Eq. (24) can be written in an alternate form without changing the result if the last term of each sum is treated separately. Then, we get:

$$
\sum_{i=1}^{n}\left|F_{i}\right| \cdot F_{n}-\sum_{i=1}^{n}\left|F_{i}\right| \cdot F_{i}=\left\{\sum_{i=1}^{n-1}\left|F_{i}\right| \cdot F_{n}+\left|F_{n}\right| \cdot F_{n}\right\}-\left\{\sum_{i=1}^{n-1}\left|F_{i}\right| \cdot F_{i}+\left|F_{n}\right| \cdot F_{n}\right\}
$$

The right-hand side of Eq. (25a) can be simplified and we get the following relationship:

$$
\sum_{i=1}^{n}\left|F_{i}\right| \cdot F_{n}-\sum_{i=1}^{n}\left|F_{i}\right| \cdot F_{i}=\sum_{i=1}^{n-1}\left|F_{i}\right| \cdot F_{n}-\sum_{i=1}^{n-1}\left|F_{i}\right| \cdot F_{i}
$$

In the next step, after replacing the difference between the two single sums on the right-hand side of Eq. (24) with the right-hand side of Eq. (25b), we get the following alternate version of Eq. (24):

$$
\Longrightarrow L=\sum_{i=1}^{n-1} \sum_{j=i+1}^{n} F_{i} \cdot\left|F_{j}\right|+\sum_{i=1}^{n-1} \sum_{j=1}^{i} F_{i} \cdot\left|F_{j}\right|+\underbrace{\sum_{i=1}^{n-1}\left|F_{i}\right| \cdot F_{n}-\sum_{i=1}^{n-1}\left|F_{i}\right| \cdot F_{i}}_{\text {Equation (25b) }}
$$

The second double sum on the right-hand side of Eq. (26) above can be rewritten by using a universally applicable relationship that describes how dependent indices of a double sum are to be interchanged (see Ref. [10], Section 3.5.2, Fact 8). This relationship can be expressed as follows after (i) both the sides of the original equation given in Ref. [10] and the summation indices " $i$ " and " $j$ " are switched and (ii) the symbol of the upper limit " $n$ " is replaced by the alternate generic symbol " $\psi$ ":

$$
\text { Ref. [10], Section 3.5.2, Fact } 8 \Longrightarrow \sum_{i=1}^{\psi} \sum_{j=1}^{i} \varphi(i, j)=\sum_{j=1}^{\psi} \sum_{i=j}^{\psi} \varphi(i, j)
$$

The symbol $\varphi(i, j)$ above represents a summation term that depends on two indices. Then, after applying the relationship defined in Eq. (27a) above to the second double sum on the right-hand side of Eq. (26) and after replacing the generic upper bound " $\psi$ " of the summations with " $n-1$ ", we get:

$$
\sum_{i=1}^{n-1} \sum_{j=1}^{i} F_{i} \cdot\left|F_{j}\right|=\sum_{j=1}^{n-1} \sum_{i=j}^{n-1} F_{i} \cdot\left|F_{j}\right|
$$


Indices used on the right-hand side of Eq. (27b) can be switched below the summation symbols and in the summation term definition without changing the result. Then, we get:

$$
\sum_{i=1}^{n-1} \sum_{j=1}^{i} F_{i} \cdot\left|F_{j}\right|=\sum_{i=1}^{n-1} \sum_{j=i}^{n-1} F_{j} \cdot\left|F_{i}\right|
$$

The Commutative Law can also be applied to the summation term of the double sum on the right-hand side of Eq. (27c) as $F_{j} \cdot\left|F_{i}\right|$ equals $\left|F_{i}\right| \cdot F_{j}$. Then, we get the following alternate form of Eq. (27c):

$$
\sum_{i=1}^{n-1} \sum_{j=1}^{i} F_{i} \cdot\left|F_{j}\right|=\sum_{i=1}^{n-1} \sum_{j=i}^{n-1}\left|F_{i}\right| \cdot F_{j}
$$

The left-hand side of Eq. (27d) above equals the second double sum on the right-hand side of Eq. (26). Consequently, the right-hand side of Eq. $(27 d)$ can be used to replace it. Then, we get the following alternate form of Eq. (26):

$$
\Longrightarrow L=\sum_{i=1}^{n-1} \sum_{j=i+1}^{n} F_{i} \cdot\left|F_{j}\right|+\underbrace{\sum_{i=1}^{n-1} \sum_{j=i}^{n-1}\left|F_{i}\right| \cdot F_{j}}_{\text {Equation }(27 d)}+\sum_{i=1}^{n-1}\left|F_{i}\right| \cdot F_{n}-\sum_{i=1}^{n-1}\left|F_{i}\right| \cdot F_{i}
$$

The outer summation of the last three sums on the right-hand side of Eq. (28) is identical. Therefore, the last three sums can be combined. Then, we get the following alternate form of Eq. (28):

$$
\Longrightarrow L=\sum_{i=1}^{n-1} \sum_{j=i+1}^{n} F_{i} \cdot\left|F_{j}\right|+\sum_{i=1}^{n-1}\left[\left\{\sum_{j=i}^{n-1}\left|F_{i}\right| \cdot F_{j}\right\}+\left|F_{i}\right| \cdot F_{n}-\left|F_{i}\right| \cdot F_{i}\right]
$$

The single sum inside the square brackets on the right-hand side of Eq. (29) above can be expressed in a different form after both the lower and upper bounds of the summation are shifted by one. Consequently, the following relationship applies:

$$
\sum_{j=i}^{n-1}\left|F_{i}\right| \cdot F_{j}=\left\{\sum_{j=i+1}^{n}\left|F_{i}\right| \cdot F_{j}\right\}-\left|F_{i}\right| \cdot F_{n}+\left|F_{i}\right| \cdot F_{i}
$$

Then, after solving for the single sum on the right-hand side of Eq. (30), we get:

$$
\sum_{j=i+1}^{n}\left|F_{i}\right| \cdot F_{j}=\left\{\sum_{j=i}^{n-1}\left|F_{i}\right| \cdot F_{j}\right\}+\left|F_{i}\right| \cdot F_{n}-\left|F_{i}\right| \cdot F_{i}
$$

The right-hand side of Eq. (31) matches all terms that are given inside the square brackets on the right-hand side of Eq. (29). Therefore, the left-hand side of Eq. (31) can be used to replace the contents of the square brackets on the right-hand side of Eq. (29). Then, Eq. (29) takes on the following form:

$$
\Longrightarrow L=\sum_{i=1}^{n-1} \sum_{j=i+1}^{n} F_{i} \cdot\left|F_{j}\right|+\sum_{i=1}^{n-1} \underbrace{\sum_{j=i+1}^{n}\left|F_{i}\right| \cdot F_{j}}_{\text {Equation (31) }}
$$

The right-hand side of Eq. (32) above matches the right-hand side of Eq. (20) which completes the proof of Eq. (20). In addition, we know that Eq. (20) equals Eq. (18). Therefore, the validity of Eq. (17) was 
rigorously proven as Eq. (17) and Eq. (18) only differ by the fact that each summation term is multiplied by a constant.

It must be mentioned that the individual regression model terms on the left- and right-hand sides of Eq. (17) match but the order of the summation differs. Therefore, users of the Iterative Method should continue to use the two traditional term groups on the right-hand side of Eq. (17) for the regression model definition of an electrical output even though (i) it is the more complicated way of describing all possible combinations of $F_{i}$ and $\left|F_{j}\right|$ and (ii) hides the fact that the two term groups $F_{i} \cdot\left|F_{j}\right|$ and $\left|F_{i}\right| \cdot F_{j}$ must either be used together or must both be omitted. This approach guarantees compatibility with the term order used in many wind tunnel facilities that implemented the Iterative Method as recommended by AIAA's IBTWG (see Ref. [2], Eq. (3.1.3)). However, any new implementation of the Non-Iterative Method in the data system of a wind tunnel can take advantage of the simplicity of the left-hand side of Eq. (17) for the definition of the regression model term groups of a balance load as no industry-wide "standard" for implementation \& use of the Non-Iterative Method currently exists.

Again, it is important to emphasize that the rigorous proof of Eq. (17) lead to the conclusion that the traditionally used regression model term groups $F_{i} \cdot\left|F_{j}\right|$ and $\left|F_{i}\right| \cdot F_{j}$ belong to the single group. Therefore, they must either be used together or must both be omitted if an analyst uses the regression model definition of Ref. [2] for the description of bi-directional output characteristics. 